\title{
Isolated DC and AC Current Amplifier with Magnetic Field Sensor in Loop and Amorphous Ring Core
}

\author{
O. Petruk ${ }^{a}$, M. Kachniarz ${ }^{a, *}$ And R. SzeWCZyK ${ }^{b}$ \\ ${ }^{a}$ Industrial Research Institute for Automation and Measurements, Al. Jerozolimskie 202, 02-486 Warsaw, Poland \\ ${ }^{b}$ Institute of Metrology and Biomedical Engineering, Warsaw University of Technology, \\ sw. Andrzeja Boboli 8, 02-525 Warsaw, Poland
}

\begin{abstract}
This paper presents innovative isolated DC and AC current amplifier containing magnetic field sensor in the feedback loop. The amorphous ring core with an air gap is utilized as a part of the galvanic isolation separating input and output currents. In the paper outline of the amplifier is presented. The printed circuit board project was developed and electronic circuit of the amplifier was manufactured. The developed device was investigated with $\mathrm{DC}$ and $\mathrm{AC}$ current and the results are presented in the paper. Results indicate usefulness of the developed device in described application.
\end{abstract}

DOI: 10.12693/APhysPolA.131.1174

PACS/topics: 85.75.Ss, 07.55.Ge, 85.30.Fg

\section{Introduction}

Measurement of the leakage current in the power lines overvoltage protection is a very important issue from the point of view of the energy industry. This kind of measurement requires isolated measurement system for current measurement. Practical implementation of such measurement system is difficult, because it is necessary to ensure galvanic isolation for several kilovolts.

For this kind of measurements, special isolated current amplifier was developed. Previously, many approaches to the design of current amplifier were made, like utilization of mesoscopic Josephson junction [1] or cryogenic current comparator [2]. The most important group of current amplifiers are devices based on the CMOS technology [35].

The developed current amplifier contains the Halleffect sensor [6] of magnetic field connected in the feedback loop. Magnetic field measured by the sensor is generated by the magnetized amorphous ring core with an air gap [7]. Magnetic core also provides galvanic isolation between the primary and secondary windings. The developed device is similar to the classical current transformer with magnetic ring core and magnetic field sensor $[8,9]$. However, unlike the traditional current transformer, the presented construction allows to increase the value of the input current, which is very important in measurements of very low currents, like leakage currents in surge arresters [10]. In the presented solution it is possible to measure low current of about several $\mu \mathrm{A}$ at the voltage value of up to several $\mathrm{kV}$.

\section{Outline of the developed current amplifier}

The principle of operation of the presented device is similar to the classical current transformer. The main

*corresponding author; e-mail: mkachniarz@piap.pl

component is magnetic ring core with an air gap. Magnetic field sensor is placed inside the air gap. Schematic block diagram of the developed device is presented in Fig. 1.

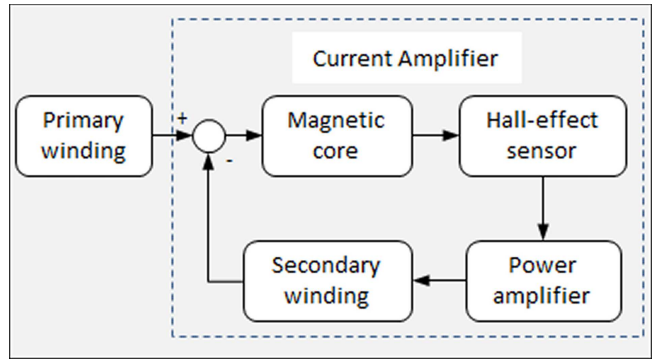

Fig. 1. Schematic block diagram of the developed isolated current amplifier.

On the toroidal magnetic core two sets of windings are made: primary and secondary (compensating). Input current $I_{i n}$ is applied to the primary winding. Its presence results in appearance of magnetic flux density $B_{\text {in }}$ within the core according to the equation

$$
B_{\text {in }}=\mu_{0} \mu H=\mu_{0} \mu \frac{n_{p} I_{i n}}{l},
$$

where $\mu_{0}$ is magnetic permeability of free space, $\mu$ is magnetic permeability of the core's material, $H$ is magnetizing field produced by primary winding, $n_{p}$ is number of turns in primary winding and $l$ is flow path of magnetic flux in the core. Magnetic flux density $B_{e x}$ in the air gap could be approximated with the equation [11]:

$$
B_{e x}=\mu_{0} \frac{n_{p} I_{i n}}{l_{g}}-\frac{l-l_{g}}{l_{g}} B_{i n},
$$

where $l_{g}$ is length of the gap. For magnetic cores exhibiting high permeability $\mu$ Eq. (2) can be written as

$$
B_{e x}=-\frac{l-l_{g}}{l_{g}} B_{i n} .
$$

The resulting magnetic flux density $B_{e x}$ is proportional to the input current $I_{i n}$. As a magnetic core for pre- 
sented device nanocrystalline material with permeability $\mu=8000$ was used, thus Eq. (3) is correct for this case.

Measurement of the magnetic flux density $B_{e x}$ in the air gap is performed with the Hall-effect sensor. For presented application TOSHIBA THS119 sensor was chosen, made of gallium arsenide (GaAs) and characterized by current-related sensitivity of about $20 \mathrm{~V} / \mathrm{AT}$.

The output signal from the sensor (the Hall voltage) is delivered to the input of power amplifier and then to the secondary (compensating) winding. Magnetic field in the secondary winding is opposite to the magnetizing field produced by the primary winding. Feedback loop connection allows to compensate the magnetizing field by forcing suitable current flow in the secondary winding. The result is reduction of magnetic flux density $B_{e x}$ in the air gap almost to zero. If the number of turns in secondary winding is lower than in primary one, the compensating current will be higher than input current $I_{\text {in }}$ so the compensating current can be treated as an amplified current, which is the output parameter of the developed current amplifier. The compensating output current $I_{\text {out }}$ is proportional to the input current $I_{i n}$ and ratio of primary $n_{p}$ to secondary $n_{s}$ windings

$$
I_{\text {out }}=\frac{n_{p}}{n_{s}} I_{\text {in }} .
$$

In the developed device there is 1000 turns of the primary winding and 10 turns of the secondary winding, which according to Eq. (4) results in gain factor of 100 . Modification of the gain factor is possible by changing the ratio of primary to secondary windings.

Prototype of the amplifier was developed according to the presented description. Photo of the complete device is presented in Fig. 2.

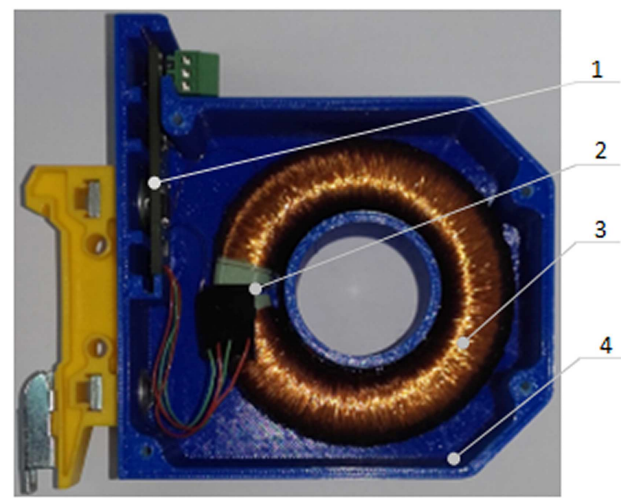

Fig. 2. Photo of the developed isolated current amplifier, 1 - electronic module, 2 - air gap with Halleffect sensor, 3 - magnetic amorphous core with 1000 primary and 10 secondary windings (secondary under primary), 4 - housing.

The printed circuit board (PCB) was designed and manufactured for electronic part of the device. The Halleffect sensor was mounted in the air gap of the nanocrystalline core. The device was mounted in the specially developed housing.

\section{Measurement stand}

The developed isolated current amplifier was investigated on the special measurement stand. Schematic block diagram of the stand is presented in Fig. 3 .

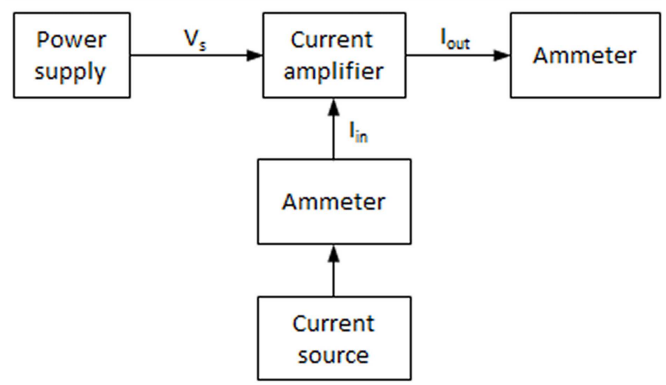

Fig. 3. Schematic block diagram of the measurement stand.

The presented device requires symmetrical voltage supply $\pm 15 \mathrm{~V}$, which was provided with the laboratory stabilized power supply. The input current $I_{i n}$ was generated with INMEL 60 industrial calibrator working in the mode of current source. The device allows to obtain current value within the range from 0 to $20 \mathrm{~mA}$, but during the measurement maximum applied current was $1.20 \mathrm{~mA}$. Input current $I_{\text {in }}$ was measured with precise FLUKE 8808A multimeter and delivered to the input of the developed current amplifier. The output current $I_{\text {out }}$ was measured with another FLUKE 8808A device.

\section{Experimental results}

Developed isolated current amplifier was investigated on the described measurement setup. Characteristic of the dependence between input and output current was measured with the input current range from 0 to $1.20 \mathrm{~mA}$. Obtained results are presented in Fig. 4. As it can be seen, the developed current amplifier exhibits very linear characteristic within entire investigated range. Coefficient of linear determination $R^{2}$ of the characteristic is 0.999 which confirms high linearity of obtained results. Obtained values of output current $I_{\text {out }}$ are slightly different than expected values $I_{\text {out }}^{\prime}$, which is also visible in the equation presented in Fig. 4. For each tested value of input current error of the amplifier was calculated according to the equation

$$
\Delta=\frac{I_{\text {out }}-I_{\text {out }}^{\prime}}{I_{\text {out }}} 100 \%=\frac{I_{\text {out }}-100 \cdot I_{\text {in }}}{I_{\text {out }}} 100 \% .
$$

The results of this calculation are presented in Fig. 5.

For low values of input current error of the developed amplifier is relatively high (over $11 \%$ for $I_{\text {in }}=19.2 \mu \mathrm{A}$ ). With the increase of the input current error is exponentially decreasing and obtains value $0.35 \%$ for input current $I_{i n}=1.0 \mathrm{~mA}$. The error of the amplifier is resulting from hysteresis of the magnetic core and offset voltage of the Hall-effect sensor, which influences the value of output current. 


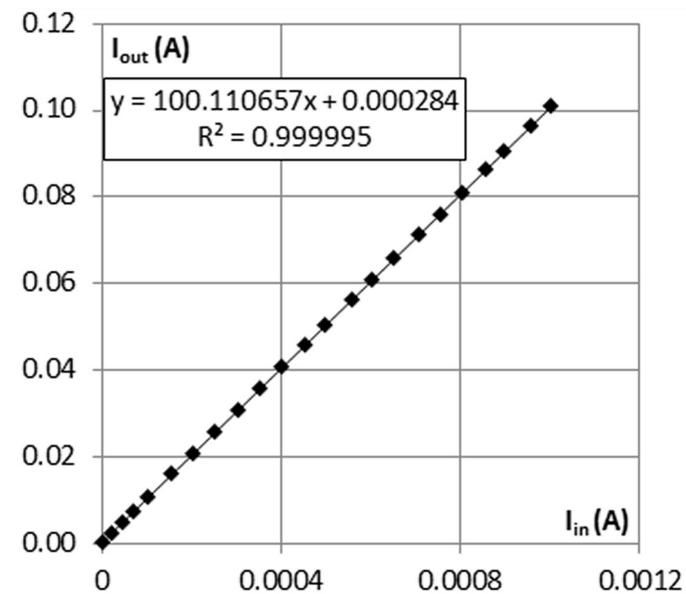

Fig. 4. Characteristic of relation between output and input current of the developed current amplifier.

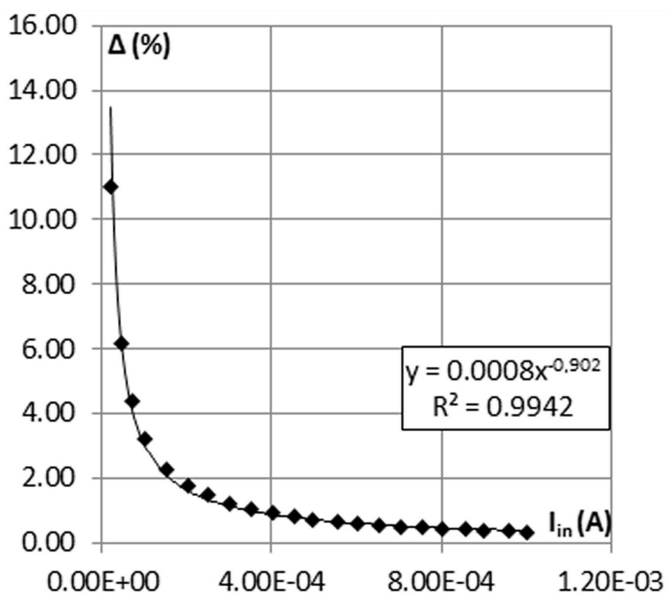

Fig. 5. Input current dependence of the error of developed current amplifier.

\section{Conclusions}

The developed current amplifier presented in the paper is working correctly. Obtained results are close to the expected ones, which is confirmed by low values of the error, especially for the higher input currents. The device exhibits high linearity of the characteristic. Gain factor of 100 allows measurement of the very low currents, even several $\mu \mathrm{A}$. Galvanic isolation provided by the magnetic core enables to perform measurement at the voltage value reaching even several $\mathrm{kV}$. The device can work with $\mathrm{DC}$ currents as well as with AC currents of frequency not exceeding $5 \mathrm{~Hz}$ (for higher frequencies the amplifier tends to oscillate). The developed isolated current amplifier is a new approach to the classic construction of the DC current transformer.

\section{Acknowledgments}

This work was partially supported by the statutory founds of Institute of Metrology and Biomedical Engineering, Warsaw University of Technology (Poland).

\section{References}

[1] J. Delahaye, J. Hassel, R. Lindell, M. Sillanpää, M. Paalanen, H. Seppä, P. Hakonen, Science 299, 1045 (2003)

[2] F. Gay, F. Piquemal, G. Genevès, Rev. Sci. Instrum. 71, 4592 (2000).

[3] C.Y. Wu, W.M. Chen, L.T. Kuo, IEEE Trans. Biomed. Circuits Syst. 7, 107 (2013).

[4] G. Di Cataldo, R. Mita, S. Pennisi, Microelectron. J. 37, 1086 (2006).

[5] G. Palmisano, G. Palumbo, S. Pennisi, CMOS Current Amplifiers, Springer Science + Business Media, New York 1999.

[6] E. Ramsden, Hall-Effect Sensor: Theory and Application, Newnes, Oxford 2006.

[7] J. Salach, A. Bieńkowski, R. Szewczyk, J. Magn. Magn. Mater. 316, E607 (2007).

[8] P. Ripka, Magnetic Sensors and Magnetometers, Artech, Boston 2001.

[9] O. Petruk, R. Szewczyk, J. Salach, M. Nowicki, in: Advances in Intelligent Systems and Computing, Vol. 267, Eds. R. Szewczyk, C. Zieliński, M. Kaliczyńska, Springer, Berlin 2014, p. 641.

[10] J. Salach, L. Hasse, R. Szewczyk, J. Smulko, A. Bieńkowski, P. Frydrych, A. Kolano-Burian, IEEE Trans. Magn. 48, 1493 (2012).

[11] D.C. Jiles, Introduction to Magnetism and Magnetic Materials, Chapman \& Hall/CRC, London 1998. 